\title{
ANÁLISE DAS DOSAGENS DE TIROXINA (T4) EM PACIENTES COM HIPOTIREOIDISMO NA REGIÃO DA ASA NORTE - BRASÍLIA, DF, BRASIL
}

\author{
Alvaro Carlos Galdos-Riveros ${ }^{1}$; Allan Viana Costa ${ }^{2}$, Luciana do Nascimento Reis ${ }^{2}$ \\ ${ }^{1}$ Professor Doutor do Instituto de Ciências da Saúde, Universidade Federal de Mato \\ Grosso, Sinop (alvarogaldos@usp.br) \\ ${ }^{2}$ Farmacêutico, Faculdade Anhanguera de Brasília
}

Recebido em: 08/04/2017 - Aprovado em: 10/06/2017 - Publicado em: 20/06/2017

DOI: 10.18677/EnciBio_2017A121

\begin{abstract}
RESUMO
A glândula da tireoide é responsável pela produção de hormônios, que é de estrema importância para o desenvolvimento do ser humano, localiza-se abaixo da laringe na parte anterior da traqueia, possui um formato de borboleta com dois lobos um direito e um esquerdo ligado pelo istmo. A desregulação dessa glândula causa o hipotireoidismo que é a pouca liberação dos hormônios da tireoide, sendo tratada com tiroxina (T4) e triiodotironina (T3). O objetivo deste trabalho foi analisar as dosagens de T4 em diferentes faixas etárias em pacientes com hipotireoidismo na região da Asa Norte - DF, durante o ano de 2015, sendo analisado: idade, sexo e dosagens de T4. Os critérios de inclusão foram pacientes com diagnóstico de hipotireoidismo, que fizeram administração de T4 sendo realizada uma análise descritiva dos dados. Os dados mais relevantes da pesquisa foram que a maioria dos problemas da tireoide ocorreram em mulheres com idades entre 41 e 50 , e as dosagens mais usadas são as de $25 \mu \mathrm{g}$. Pode-se concluir baseados nos resultados, que se houvesse uma atenção maior a essa doença e um incentivo em pesquisas conseguiriam diagnosticar precocemente a mesma, evitando tantos casos de hipotireoidismo no Brasil.
\end{abstract}

PALAVRAS-CHAVE: Células foliculares, Nódulos da tireoide, Triiodotironina(T3).

\section{ANALYSIS OF THYROXINE (T4) DOSE IN PATIENTS WITH HYPOTHYROIDISM IN THE REGION OF ASA NORTE - DISTRITO FEDERAL, BRASIL}

\footnotetext{
ABSTRACT

The thyroid gland is responsible for producing hormones, which is of extreme importance for the development of human beings, it is located below the larynx in the anterior part of the trachea, and it has a butterfly-shaped with two lobes, one right and left bound by the isthmus. The gland dysfunction causes Hypothyroidism that is a short release of thyroid hormones, being treated thyroxine (T4) and triiodothyronine (T3). The objective of this study was to analyze the T4 dosages in different age groups of patients with hypothyroidism in the region at Asa Norte - DF, during the year 2015, analyzing age, sex and T4 dosages. The Inclusion parameter was made using patients diagnosed with hypothyroidism, which made administration of T4 being
} 
performed an analysis descriptive data. The most relevant data from the survey were that most of thyroid problems occurred in women aged 41 and 50, and more dosages used are the $25 \mathrm{mcg}$. We can conclude based on results, that if there were more attention to this disease and an incentive research would get early diagnoses, avoiding many cases of hypothyroidism in Brasil.

KEYWORDS: Triiodothyronine (T3), Nodules of the thyroid, Follicular cells.

\section{INTRODUÇÃO}

As disfunções da tireoide acometem em média $12 \%$ da população geral, segundo dados do Arquivo Brasileiro de Endocrinologia e Metabologia. Cerca de $4,6 \%$ da população dos Estados Unidos possui hipotireoidismo primário, sendo a maioria mulheres e idosos (SILVA, 2005). A tireoide vem do grego thyreos que significa escudo, tem forma de borboleta, localizando-se abaixo da laringe na parte anterior da traqueia, com um peso entre 15 e 25 gramas em adultos. Tem um amplo suprimento de sangue que saem das artérias tireocervicais e do sistema nervoso autônomo, secretando T4 e T3 (WHALEN et al., 2016).

O hipotálamo e a hipófase regulam os hormônios da tireoide. O hormônio estimulante da tireoide (TSH) da hipófise anterior eleva a liberação de tiroxina e triiodotironina, sendo secretado e controlado pelo hormônio do hipotálamo $(T R H)$. O $\mathrm{TRH}$ é levado do sangue hipotalâmico para o hipofisário, afetando as células hipofisárias anterior, aumentando com isso o TSH, se ocorre um bloqueio nesse sistema a taxa de liberação de TSH é reduzida pela hipófise anterior (HALL, 2011).

A tireoide é constituída de várias células foliculares possuindo uma substância central chamada coloide, responsável por secretar dois hormônios, triiodotironina (T3) e tiroxina (T4), sob a ação reguladora hipofisária do TSH, que obedece ao comando hipotalâmico do homônimo liberador de tropina (GUEDES et al., 2006). O valor de referência de TSH sérico normal para adultos é entre 0,4 - 4,5 mUI/D (CARVALHO et al., 2013).

A T4 é produzida em maior quantidade do que a T3 e é formada na porção amina-terminal da tiroglobulina enquanto a T3 é formada por porção carboxiterminal, podendo também a T4 se transformar em T3 através da desiodação da T4 dependendo do estímulo da TSH (LONGO et al., 2013). As principais disfunções da tireoide são: hipertireoidismo e hipotireoidismo. $O$ hipertireoidismo acomete pacientes com excesso de hormônio associado a várias doenças, podendo ser tratada através de extração de tecido tireoidiano, irradiação de iodo, cirurgia ou fármacos (GROSSMAN \& PORTH, 2016).

O hipotireoidismo é uma redução dos hormônios tireoideos diminuindo a ação metabólica podendo ser congênito ou adquirido. O hipotireoidismo congênito só ocorre se não for corrigido precocemente, já o hipotireoidismo adquirido ocorre por causas primárias ou secundárias. No primário ocorre remoção cirúrgica total ou parcial da tireoide. No secundário pode surgir o aumento de iodo ou disfunção da hipófise, sendo induzida por alguns medicamentos (HALL, 2011; LONGO et al., 2013).

O tratamento do hipotireoidismo é feito com a ingesta de alguns fármacos como a levotiroxina sódica, que é um sal sódico do isômero levorrotatório da T4, é absorvida pelo trato gastrointestinal com uma meia vida de sete dias, sendo administrada uma vez ao dia (LONGO et al., 2013). Diante do exposto, é de grande relevância o estudo das dosagens de T4 e a dinâmica das mudanças na dosagem em pacientes com faixa de idade distinta, sendo de muita importância na saúde pública. O objetivo deste estudo foi analisar, com base nas prescrições, as dosagens 
de tiroxina (T4), em pacientes com hipotireoidismo na região da Asa Norte - DF.

\section{MATERIAL E MÉTODOS}

Este estudo é uma pesquisa do tipo experimental que consistiu na coleta de dados provenientes de receitas de farmácia nos meses de fevereiro a outubro de 2015. Para a construção dos conhecimentos foram utilizadas informações provenientes de artigos, livros e sites específicos da área no ano de 2015. Foram utilizadas as seguintes fontes de dados: Pubmed, Scielo, Bireme, Google acadêmico com as seguintes palavras-chave: hipotireoidismo, triiodotironina (T3) e tiroxina (T4), tireoide, nódulos na tireoide e células foliculares.

Esta pesquisa realizou uma amostragem de 255 receitas de uma farmácia localizada na Região da Asa Norte - DF nos meses de fevereiro a outubro de 2015, tratando-se de um estudo que avaliou somente receitas, portanto não exigiu comitê de ética em pesquisa (Plataforma Brasil).

Os critérios de inclusão foram pacientes com diagnóstico de hipotireoidismo, que fazem administração de T4, sendo realizada uma análise descritiva dos dados, através de tabelas de frequência. Os dados mais relevantes serão mostrados através de gráficos de colunas, sendo feita uma comparação entre mulheres e homens.

\section{RESULTADOS E DISCUSSÃO}

O hipotireoidismo é uma doença de múltiplas etiologias que apresentam um elevado acervo de sinais e sintomas que vão desde alterações irreversíveis do desenvolvimento em idades precoces até manifestações clínicas inespecíficas e insidiosas em indivíduos acima de 18 anos, que podem afetar sua qualidade de vida. Além disso, o hipotireoidismo associa-se a co-morbilidades que incluem alterações no metabolismo dos carboidratos e lipídios, cardiopatias, infertilidade, alterações hematológicas, neurológicas e transtornos do ânimo (CHAKERA et al., 2012; GARBER et al., 2012; BRENTA et al., 2013; CENTANNI, 2013).

O presente estudo permitiu documentar os padrões de prescrição do hormônio tireoide e as doses empregadas com maior frequência em pacientes diagnosticados na região norte de Brasília, DF - Brasil. Os resultados demonstraram que poderiam serem úteis em futuras decisões na escolha de tratamentos com maior qualidade e atenção aos pacientes com hipotireoidismo.

Os resultados demonstraram que existem diferenças significativas na idade, sexo e dosagem das prescrições do hormônio da tireoide, em especial porque os dados mostram que as mulheres a utilizam com maior frequência do que os homens (F: 82\%; M: 18\%) e em menores de 20 anos apresentou 5,9\% de casos.

Os resultados desta pesquisa corroboram estudos desenvolvidos em vários países (ASOCIACIÓN COLOMBIANA DE ENDOCRINOLOGÍA, 1999; GARBER et al., 2012; BRENTA et al., 2013; ESCRIBANO-SERRANO et al., 2016). Essa análise foi realizada em uma farmácia na Região da Asa Norte-DF, sendo coletadas 255 receitas de pacientes com hipotireoidismo provenientes de diferentes planos de saúde tanto público como privado. Os resultados mostraram que a distribuição por sexo mostrou que 209 pacientes (82\%) foram mulheres. Na distribuição por idades, $5,9 \%$ eram menores de 20 anos, pacientes mulheres com idades de $41-50$ apresentaram o maior índice de prescrições $(47,4 \%)$ e a dosagem que prevaleceu nas mulheres foram a de $25 \mu \mathrm{g}$, enquanto que em homens a maior incidência foi entre $51-60$ anos (28\%) com dosagens de 25 e $30 \mu \mathrm{g}$.

A apresentação do medicamento prescrito na farmácia foi a Levotiroxina 
(99,9\%), similar em países como o Reino Unido, onde a utilizam o $99,7 \%$ dos pacientes (MITCHELL et al., 2009), na Colômbia (MACHADO-ALBA et al., 2013). A Levotiroxina sódica mais vendida comercialmente apresenta as seguintes dosagens: 25, 50, 75, 88 e $100 \mu \mathrm{g}$ cartuchos de 28 comprimidos (BULARIUM, 2013).

Estudos realizados com 3.567 testes de TSH no Laboratório de Análises Clínicas Oswaldo Cruz do município de Juína no Mato Grosso no período de janeiro de 2009 a dezembro de 2013, mostraram que 77,9\% (2.779) eram mulheres e 22,1\% (788) homens, havendo um aumento de $487,87 \%$ no número de solicitações de TSH depois de algum tempo.

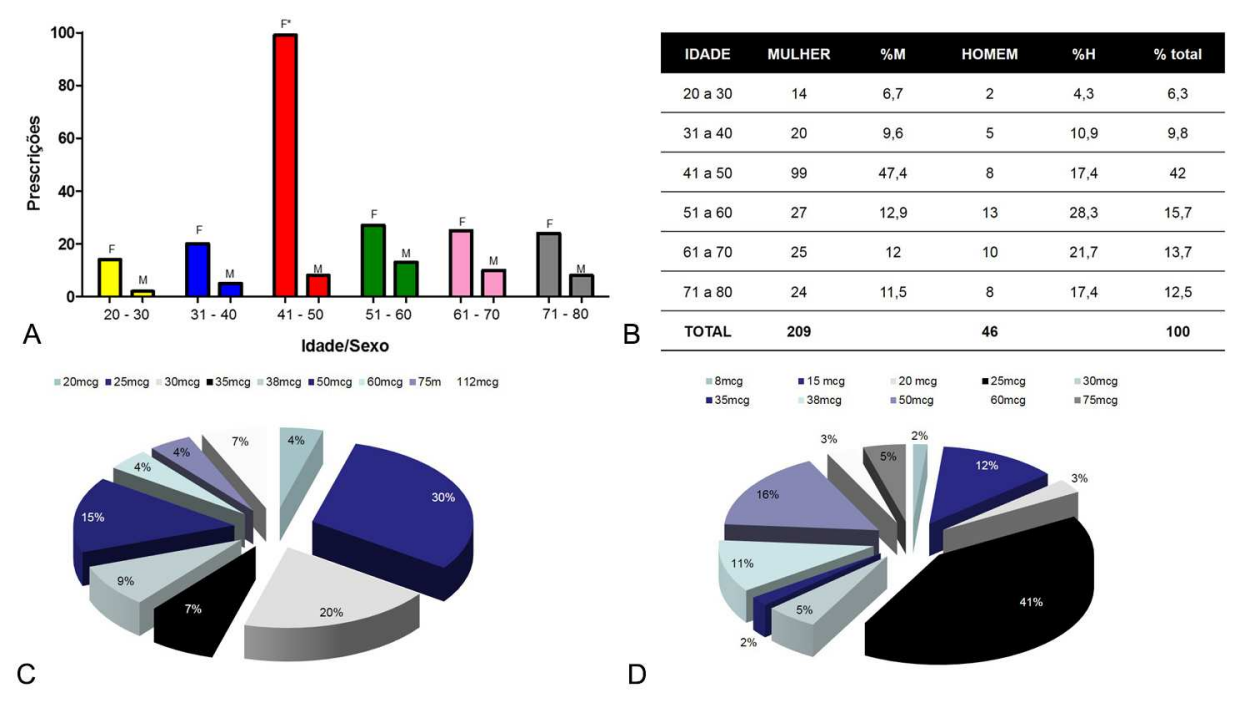

FIGURA 1 - Dados obtidos na pesquisa sob Tiroxina em pacientes com hipotireoidismo. Podemos observar: $\operatorname{Em}(A)$ que a faixa etária com maior pedido de Levotiroxina sódica foi entre 41 - 50, já em (B) encontramos as principais porcentagens referentes a idade e sexo na utilização do medicamento na farmácia. Em (C) o gráfico mostra a dosagem mais consumida por homens, que foi de 25 e $30 \mu \mathrm{g}$ e em (D) a dosagem em mulheres com maior incidência foi de $25 \mu \mathrm{g}$. Fonte: Própria.

De acordo com PEDROSO \& TANEDA (2015) os casos confirmados de hipotireoidismo 226 (78\%) foram mulheres e 63 (22\%) homens numa cidade de Mato Grosso. Sendo que as idades que mais prevaleceram nas mulheres foram 41 a 50 anos e nos homens de 51 a 60 anos. Os resultados desta pesquisa também mostraram a mesma faixa atingida pela doença.

Relatos de MARROCO \& KLOSS (2002) mostraram que 5\% das mulheres com idade entre 40 e 60 anos e $7 \%$ das mulheres com mais de 60 anos apresentaram o hipotireoidismo, sendo que esse número sobe para $20 \%$ em mulheres que tenham entre 70 a 80 anos.

Em um estudo realizado com 2.594 pacientes, 314 apresentaram hipotireoidismo, sendo que 68 (22\%) eram homens e 246 (78\%) eram mulheres, 0 que sugere uma maior prevalência deste gênero nesta patologia. Destas mulheres, 20 tinham menos de 50 anos e 226 estavam acima dessa idade (BANDONI, 2011), o que indica que a faixa etária elevada possa ser um fator importante já que nas mulheres apresentam início na menopausa.

De acordo com SICHIERI et al. (2007) em 1.298 mulheres da cidade do Rio de Janeiro, encontraram $12,3 \%$ de alteração de TSH desta população. Também avaliaram essa prevalência em três grupos étnicos, e alcançaram o resultado de 
$6,9 \%$ entre mulheres negras, $8,8 \%$ entre as mulatas e $16,7 \%$ entre as mulheres brancas. Enquanto que os resultados desta pesquisa apontam alteração nos níveis de TSH nos pacientes com hipotireoidismo utilizados nesta pesquisa, uma vez que eles são paciente diagnosticados.

$\mathrm{Na}$ Inglaterra ocorreu a prevalência de hipotireoidismo em $7,5 \%$ no sexo feminino e $3 \%$ no sexo masculino. Enquanto que em um estudo conduzido no Colorado, Estados Unidos com 25.862 indivíduos foi encontrado 9,5\% de TSH alterado sendo que $20 \%$ dessas alterações foram em população idosa (CANARIS et al.,2000).

Os medicamentos mais usados na associação com levotiroxina sódica foram os anti-ulcerosos e sais de ferro, cálcio e alumínio (60\%), que com o uso concomitante de outros medicamentos e alimentos podem interferir na farmacocinética dos hormônios tireoidianos (CENTANNI, 2013).

\section{CONCLUSÃO}

Neste trabalho foi abordado o assunto sobre a doença da tireoide (hipotireoidismo) e seu tratamento, fazendo uma revisão de um melhor tratamento com a tiroxina (T4). Portanto, conclui-se que são necessárias medidas tanto de atenção, diagnóstico e prevenção da doença, baseadas nas pesquisas desenvolvidas tanto na região estudada assim como de outras regiões do Brasil.

\section{REFERÊNCIAS}

ASOCIACIÓN COLOMBIANA DE ENDOCRINOLOGÍA. Consenso colombiano para el diagnóstico y manejo de las enfermedades tiroideas. Acta Médica Colombiana., v. 24, n. 4, 159-174, 1999. Disponível em: < http://www.actamedicacolombiana.com/anexo/articulos/04-1999-06.pdf>. Acesso em: 4 Jan 2017.

BANDONI, T. R. F. F. Hipotireoidismo subclínico: incidência de casos em mulheres da região do estado de São Paulo, Labmaricondi, v. 1, n. 1, p. 1-22, 2011. Disponível em: < http://www.labmaricondi.com.br/pdf/hip.pdf> Acesso em: 3 de nov 2016.

BRENTA, G.; VAISMAN, M.; SGARBI, J. A.; BERGOGLIO, L. M.; DE ANDRADA, N. C.; PINEDA, P. Clinical practice guidelines for the management of hypothyroidism. Arquivo Brasileiro de Endocrinologia Metabólica., v. 57, n. 4, p. 265-291, 2013. DOI: http://dx.doi.org/10.1590/S0004-27302013000400003

BULARIUM, São Paulo: A.C. Farmacêutica, 2013, 400p

CANARIS, G. J.; MANOWITZ, N. R.; MAYOR, G.; RIDGWAY, E. CH. The Colorado Thyroid Disease Prevalence Study. Archives of Internal Medicine., v. 160, n. 4, p. 526-534, 2000. DOI: 10.1001/archinte.160.4.526

CARVALHO, G. A.; PEREZ, C. L. S.; WARD, L. S. Utilização dos testes de função tireoidiana na prática clínica. Arquivo Brasileiro de Endocrinologia Metabólica., v. 57, n. 3, p. 193-204, 2013. Disponível em: http://www.scielo.br/pdf/abem/v57n3/v57n3a05.pdf . Acesso: 15 Set 2016.

CENTANNI, M. Thyroxine treatment: absorption, malabsorption, and novel therapeutic approaches. Endocrine., v. 43, n. 1, p. 8-9, 2013. DOI: 10.1007/s12020012-9814-9 
CHAKERA, A. J.; PEARCE, S. H.; VAIDYA, B. Treatment for primary hypothyroidism: current approaches and future possibilities. Drug design, Development and Therapy., v. 6, p. 1-11, 2012. DOI: 10.2147/DDDT.S12894

ESCRIBANO-SERRANO, J.; MANCERA-ROMERO, J.; SANTOS-SÁNCHEZ, V.; PAYÁ-GINER, C.; MÉNDEZ-ESTEBAN, M. I.; GARCÍA-BONILLA, A.; et al.,; A Prevalencia de hipotiroidismo en Andalucía según el consumo de hormona tiroidea en 2014. Revista Española de Salud Pública, v. 90, n. e40026, p. e1-e12, 2016. Disponível em: http://scielo.isciii.es/scielo.php?script=sci_arttext\&pid=S1135$57272016000100427 \&$ Ing=es\&tlng=es. Acesso em 25 dezembro 2016.

GARBER, J. R.; COBIN, R. H.; GHARIB, H.; HENNESSEY, J. V.; KLEIN, I.; MECHANICK, et al., A. Clinical practice guidelines for hypothyroidism in adults: cosponsored by the American Association of Clinical Endocrinologists and the American Thyroid Association. Endocrine Practice., v. 18, n. 6, p. 988-1028, 2012. DOI: http://dx.doi.org/10.4158/EP12280.GL

GROSSMAN, S.; PORTH, C. M. Porth Fisiopatologia. 9ªd. Rio de Janeiro: Guanabara Koogan, 2016, 1655p.

GUEDES, E. P.; MOREIRA, R. O.; BENCHIMOL, A. K. Endocrinologia. Ed. Rio de Janeiro: Rubio. 2006, 495 p.

HALL J. E. Guyton \& Hall Fundamentos de Fisiologia. 12a ${ }^{\mathrm{a}}$ Ed. Rio de Janeiro: Elsevier, 2011, 728p.

LONGO, D. L.; FAUCI, A. S.; KASPER, D. L.; HAUSER, S. L.; JAMESON, J. L.; LOSCALZO, J. Manual de Medicina de Harrison. 18ํㅡ. Porto Alegre: Artmed. 2013, 1548p.

MACHADO-ALBA, J. E.; ALZATE-CARVAJAL, V.; ECHEVERRI-CATANO, L. Estudio farmacoepidemiológico de uso de anti-inflamatórios no esteroideos en pacientes de alto riesgo cardiovascular. Revista Peruana de Medicina Experimental de Salud Pública [online]., v. 30, n.4, p. 626-629, 2013.

MITCHELL, A. L.; HICKEY, B.; HICKEY, J. L.; PEARCE, S. H. Trends in thyroid hormone prescribing and consumption in the UK. BioMedCentral. Public Health., v. 9, n. 132, p. 1-9, 2009. DOI: 10.1186/1471-2458-9-132.

MOROCCO, M.; KLOSS, M. R. T. Subclinical hypothyroidism in women: who to treat. Disease a Month., v. 48, p. 659 - 670, 2002. DOI: http://dx.doi.org/10.1053/cjwh.2000.19079

PEDROSO, B.; TANEDA, M. Análise dos Casos de Hipotireoidismo na Região Noroeste do Mato Grosso.Revista da Saúde da Ajes-Sajes, v. 1, n. 2, 2015.

PEDROSO, B.; TANEDA, M. Análise dos casos de hipotireoidismo na região noroeste do Mato Grosso. Revista de Saúde da Ajes-Sajes., v. 1, n. 1, p. 1-16, 2015. http://www.revista.ajes.edu.br/index.php/SAJES/article/view/31/pdf Acesso em: 04 out 2016. 
SICHIERI, R.; BAIMA, J.; MARANTE, T.; VASCONCELLOS, M. T. L.; MOURA, A. S.; VAISMAN, M. Low prevalence of hypothyroidism among black and mulatto people in a population-based study in Brasilian women. Clinical Endocrinology., v. 66, p. 803-807, 2007. DOI: 10.1111/j.1365-2265.2007.02816.x

SILVA, R. C; Importância da avaliação da função tireoidiana em pacientes com diabetes mellitus. São Paulo: Arquivo Brasileiro de Endocrinologia Metabólica., v. 49, n, 2, p. 2005.

WHALEN, K.; FINKEL, R.; PANAVELIL, T. A. Farmacologia llustrada, 6를. Porto Alegre: ArtMed, 2016, 670p. 